\title{
Inglês como meio de instrução na pós-graduação stricto sensu no Brasil: análise dos documentos de áreas
}

\author{
English as a medium of instruction in Brazilian graduate courses: \\ an analysis of criteria by disciplines
}

\section{Inglés como medio de instrucción en programas de posgrado en Brasil: análisis de documentos de áreas}

Telma Nunes Gimenez ${ }^{1}$

Henrique Rodrigues de Oliveira² Leonardo Amaral Carneiro ${ }^{3}$ https://orcid.org/0000-0001-6143-3895

https://orcid.org/0000-0002-4948-120X

i.ttps://orcid.org/0000-0002-1070-0604

\begin{abstract}
RESUMO: O crescimento do inglês como meio de instrução como parte de estratégias de internacionalização do ensino superior no Brasil tem sido verificado por meio de levantamentos recentes (GIMENEZ et al., 2018). Dentre as motivações para sua adoção nos programas de pósgraduação (PPGs), verifica-se o papel dos parâmetros de qualidade estabelecidos pelo sistema de avaliação da CAPES. Neste estudo, foi realizada análise de conteúdo dos documentos de áreas do conhecimento no quesito "Perspectivas do processo de internacionalização dos PPGs". Os resultados indicaram variados graus de explicitude do quesito "aulas em inglês" nas diferentes áreas do conhecimento, com predominância no Colégio de Ciências da Vida (Ciências Agrárias, Ciências Biológicas e Ciências da Saúde). Especula-se que isso se deve ao fato de essas áreas apresentarem maior poder de generalização de resultados de pesquisas e de colaboração internacional e nas quais o inglês assume papel de lingua franca da ciência com hegemonia sobre demais línguas estrangeiras.
\end{abstract}

PALAVRAS-CHAVE: Internacionalização. Pós-graduação. Inglês como meio de instrução.

\footnotetext{
${ }^{1}$ Doutora em Linguística Aplicada. Professora Sênior na Universidade Estadual de Londrina (UEL). E-mail: tgimenez@uel.br. Bolsista CNPq (Processo 311655/2018-1).

2 Estudante de graduação em Letras-Inglês, Universidade Estadual de Londrina (UEL). E-mail: henrique rodrigues2000@hotmail.com.

${ }^{3}$ Estudante de graduação em Letras-Inglês. Universidade Estadual de Londrina (UEL). E-mail: amaralc.leonardo@gmail.com.
} 
ABSTRACT: The growth of English as a means of instruction as part of the internationalization strategy for higher education in Brazil has been verified through recent surveys (GIMENEZ et al., 2018). Among the motivations for its adoption in graduate programs (PPGs) there is the role of quality parameters established by the CAPES evaluation system. In this study, a content analysis of documents from knowledge areas was carried out in the item "Perspectives of the PPG internationalization process". The results indicated varying degrees of explicitness of the item "English medium classes" in different areas of knowledge, with a predominance in the College of Life Sciences (Agrarian Sciences, Biological Sciences and Health Sciences). It is speculated that this is due to the fact that these areas have greater power to generalize research results and international collaboration and in which English functions as the lingua franca of science with hegemony over other foreign languages.

KEYWORDS: Internationalization. Graduate programs. English medium instruction.

RESUMEN El crecimiento del inglés como medio de instrucción como parte de la estrategia de internacionalización de la educación superior en Brasil se ha verificado a través de encuestas recientes (GIMENEZ et al., 2018). Entre las motivaciones para su adopción en los programas de posgrado (PPG) se encuentra el rol de los parámetros de calidad ajustados por el sistema de evaluación CAPES. En este estudio, el análisis de contenido de documentos de áreas de conocimiento se realizó en el ítem "Perspectivas del proceso de internacionalización de PPG". Los resultados indicaron diversos grados de explicitación del ítem "clases en inglés" en diferentes áreas del conocimiento, con predominio en las Ciencias de la Vida. Se especula que esto se debe a que estas áreas tienen mayor poder para generalizar resultados de investigación y colaboración internacional y en las que el inglés asume el papel de lengua franca de la ciencia con hegemonía sobre otras lenguas extranjeras.

PALABRAS CLAVE: Internacionalización. Posgraduación. Inglés como medio de instrucción.

\section{Introdução}

Em 2018, como parte de um projeto de pesquisa sobre a adoção do inglês como meio de instrução em duas universidades brasileiras ${ }^{4}$, um dos entrevistados confirma uma das motivações para passar a ofertar aulas nessa língua na pós-graduação:

\footnotetext{
Entrevistador: Você acha que, por exemplo, ofertar disciplinas em inglês atrairia mais estudantes, é isso? Por que a gente deveria pensar em ofertar?

Professor. Eu acho que... o que acontece hoje é que há uma cobrança pra isso.

Entrevistador: Da CAPES?

Professor: Da CAPES mesmo. Então as pessoas vão tentando se organizar conforme o que é cobrado.

(Entrevista, Professor de Ciências da Saúde, junho 2019)
}

\footnotetext{
4 Título do projeto: Inglês como meio de instrução em duas instituições públicas de ensino superior sob a perspectiva de Inglês como Língua Franca: política em prática.

${ }^{5}$ A CAPES - Coordenadoria de Aperfeiçoamento do Pessoal do Ensino Superior, o MEC - Ministério da Educação, tem sido um dos principais financiadores da pós-graduação no Brasil, por meio de processo de avaliação por pares.
} 
Inglês como meio de instrução na pós-graduação stricto sensu no Brasil: análise dos documentos de áreas

Esta fala atesta o impacto que agências externas podem exercer sobre essas iniciativas. A fala do professor reflete a percepção de que a "cobrança" de uma das principais fontes de financiamento da pós-graduação brasileira exerce pressão sobre a oferta ou não de disciplinas na língua estrangeira em um país onde a maioria da população fala português.

De fato, na última década, o inglês como meio de instrução (doravante IMI), tem sido adotado em ritmo crescente por instituições do ensino superior como parte de suas estratégias de internacionalização em diversas partes do mundo (DEARDEN, 2014; DOIZ; LASAGABASTER; SIERRA, 2013; MACARO et al., 2018; MURATA, 2018). Esse contexto de uso da língua tem sido definido como "[...] o uso do inglês para ensino de disciplinas acadêmicas (cujo foco não seja a própria língua inglesa) em países ou jurisdições onde a língua da maioria da população não é o inglês (DEARDEN, 2014, p. 4).

Em situações onde o inglês funciona como uma língua estrangeira, IMI geralmente tem sido adotado como forma de atrair estudantes e professores estrangeiros, bem como ampliar as possibilidades de aprendizagem e prática de inglês para os alunos (MURATA; LINO, 2018; BAUMVOL; SARMENTO, 2019a), dentre outras motivações.

As universidades brasileiras, acompanhando suas similares internacionais, estão também começando a ofertar disciplinas em inglês como parte dos esforços em desenvolver o que vem sendo chamado de internacionalização "em casa". Em 2016, um estudo publicado pelo Conselho Britânico (BRITISH COUNCIL, 2016) identificou 641 dessas disciplinas, principalmente como atividades extracurriculares. A atualização desse estudo para o período de 2017-2019 mostra um aumento para uma média de 1011 disciplinas, predominantemente em nível de pós-graduação (GIMENEZ et al., 2018). Esse rápido crescimento está acompanhado por várias sessões de treinamento de professores, ofertadas em diferentes universidades (MARTINEZ; FERNANDES, 2020; VERDU, 2017; ZÜGE; BARRETO; NOVELLI, 2020).

Apesar do aumento no número de disciplinas da graduação e da pós-graduação ministradas em inglês, pesquisas sobre esse novo contexto linguístico ainda são incipientes (BAUMVOL, 2016; BAUMVOL; SARMENTO, 2016; BAUMVOL; SARMENTO, 2019b; MARTINEZ, 2016; MARTINEZ; FERNANDES, 2020; VERDU, 2017). Entretanto, o interesse por essa área de pesquisa vem crescendo. Os títulos de quatro projetos aprovados no âmbito da chamada Brazil UK English Collaboration Cal/2018-2019, lançada 
em 2018, evidenciam esse interesse: English as a Medium of Instruction in Two Statefunded Brazilian Higher Education Institutions from a Lingua Franca Perspective: Policy in Practice, Is There a Minimum Level of English Proficiency to Teach in a University EMI Context? EMI Training for University Professors: a Potential Tool for Internationalization, e The Changing Language and Literacy Landscapes of Brazilian Universities: English in Policy Development and in Practice (BRITISH COUNCIL, 2020).

Este artigo traz uma análise de conteúdo das diretrizes de diversas áreas de conhecimento como parte do processo de avaliação da pós-graduação, consolidadas em documentos de área da CAPES, para identificar se estes mencionavam explicitamente "aulas em inglês" como quesito de qualidade. Apoiando-nos em literatura sobre IMI e internacionalização do ensino superior, buscamos discutir o modo como a língua inglesa vem se associando à internacionalização da educação superior não apenas em relação a publicações em periódicos, mas também como indicador de qualidade do ensino.

\section{IMI e a internacionalização do ensino superior}

Na última década, tem se intensificado o processo pelo qual instituições de ensino superior brasileiras buscam imprimir uma dimensão internacional às suas missões, passando a planejar estrategicamente ações para dar mais visibilidade a suas pesquisas, por meio de programas de mobilidade e/ou cooperação internacional, com vistas à melhoria de posições em rankings internacionais, dentre outras motivações (FINARDI; GUIMARÃES, 2017).

Como afirma Wit (2010), a internacionalização não se realiza da mesma maneira em todos os lugares do mundo e as estratégias são filtradas e recontextualizadas em função do contexto interno da universidade, categoria institucional e sua posição no contexto mais ampliado. Segundo ele, a crescente importância e a diversidade de entendimentos sobre o que seja internacionalização, motivações, abordagens e estratégias demandam avaliação de sua qualidade. Há pelo menos duas décadas, diversos documentos produzidos no exterior têm delineado diretrizes e instrumentos visando à avaliação da internacionalização na educação superior (Wit, 2010). Embora haja iniciativas internacionais bem sucedidas de definição de critérios e instrumentos para avaliação da qualidade da internacionalização, é amplamente reconhecido que a sensibilidade às diferenças locais é requisito essencial, tendo em vista ser este um 
Inglês como meio de instrução na pós-graduação stricto sensu no Brasil: análise dos documentos de áreas

processo fortemente influenciado pelo contexto. Esta também é a posição do European Consortium for Accreditation in Higher Education (ECA) no seu The Guide to Quality in Internationalisation (AERDEN, 2017), no qual são delineados parâmetros (frameworks) para os cursos e para as instituições.

As tensões inerentes ao propósito de situar-se no panorama global de educação superior e ser sensível às realidades locais, que também se revelam nas diferentes áreas do conhecimento, não podem ser desconsideradas em uma perspectiva crítica da internacionalização (RIZVI; LINGARD, 2010). Isto é particularmente relevante no caso das línguas, pois estas exercem papel fundamental nas trocas internacionais almejadas pelas instituições. Nesse sentido, a língua inglesa tem sido adotada como língua franca acadêmica (JENKINS, 2014; MURATA, 2018), marginalizando outras línguas, a ponto de internacionalização ser considerada sinônimo de "Englishization" (KIRKPATRICK, 2011).

O movimento que fortalece a língua inglesa é impulsionado por vários fatores, dentre eles os mecanismos de avaliação que valorizam essa língua como indicador de qualidade, como é o caso do financiamento da pós-graduação em universidades brasileiras, que será tratado na seção seguinte.

\section{A internacionalização do ensino superior e os parâmetros de qualidade da pós- graduação}

Em 2020, o presidente da CAPES informou que:

[...] 95\% das pesquisas no Brasil são feitas no âmbito da pós-graduação [...] que hoje conta com 4.649 Programas que reúnem 6.947 cursos. Destes, 3.653 são mestrados acadêmicos e 2.405 doutorados. Além disso, há também os programas profissionais com 852 mestrados e 37 doutorados. ${ }^{6}$

A criação e manutenção desses programas é feita por meio de dois processos: Avaliação das Propostas de Cursos Novos e a Avaliação dos Programas de Pós-graduação, ambos contando com a colaboração de membros da comunidade científica.

Ao longo do tempo, esse processo de avaliação tem sido orientado por parâmetros de qualidade que classificam os programas e determinam o montante de financiamento

6 CAPES. Presidente explica importância da avaliação para a pósgraduação. 2020. Disponível em: https://www1.capes.gov.br/36-noticias/10389-presidente-explica-importancia-da-avaliacao-para-a-pos-graduacao. Acesso em 10 out. 2020. 
que eles receberão da CAPES. Esse sistema é, portanto, claramente indutor da produção científica e tecnológica do país. Mais recentemente, a internacionalização passou a ocupar espaço cada vez mais relevante dentre esses parâmetros, especialmente após o término do programa Ciência sem Fronteiras ${ }^{7}$ e criação do Programa CAPES PrInt ${ }^{8}$, que instituiu novo regramento para acesso aos fundos públicos para atividades de mobilidade e cooperação internacionais.

Em dezembro de 2018, foram aprovados novos parâmetros para aferição de qualidade dos programas, com vistas a dar maior destaque à formação e avaliação de resultados do que nos processos. De acordo com notícia veiculada no site da CAPES $^{9}$ :

[...] No quesito Programa, pretende-se avaliar o funcionamento, estrutura e planejamento do programa de pós-graduação em relação ao seu perfil e seus objetivos. Quanto ao quesito Formação, a análise abrangerá aspectos como qualidade das teses, dissertações, produção intelectual de alunos e professores e das atividades de pesquisa, bem como a avaliação do egresso. Já em relação ao Impacto na Sociedade, a avaliação vai verificar o caráter inovador da produção intelectual, os efeitos econômicos e sociais do programa, internacionalização e visibilidade.

Cada área do conhecimento define os pesos para esses quesitos, a partir de limites estabelecidos pela ficha de avaliação aprovada pelo Conselho Técnico Científico da Educação Superior (BRASIL, 2020b). Assim, cada uma elaborou um Documento de Área, de estrutura similar para todas as áreas do conhecimento, no qual são contempladas suas especificidades. Na seção intitulada "Considerações sobre o futuro da área", um dos itens é "Perspectivas do processo de internacionalização dos PPGs".

\section{Metodologia do estudo}

Para identificar os indicadores de internacionalização das diferentes áreas do conhecimento e verificar se o ensino por meio do inglês constituía um requisito implícito

\footnotetext{
7 O Programa Ciência sem Fronteiras foi um "programa que busca promover a consolidação, expansão e internacionalização da ciência e tecnologia, da inovação e da competitividade brasileira por meio do intercâmbio e da mobilidade internacional" por meio de bolsas para mobilidade internacional de estudantes de graduação e pesquisadores (http://www.cienciasemfronteiras.gov.br/web/csf/o-programa).

8 O Programa CAPES PrInt sucedeu o programa Ciência sem Fronteiras, direcionando o foco para a internacionalização das universidades a partir do fomento à construção, implementação e a consolidação de planos estratégicos em áreas do conhecimento prioritárias (https://www.gov.br/capes/pt-br/acesso-a-informacao/acoes-eprogramas/bolsas/bolsas-e-auxilios-internacionais/informacoes-internacionais/programa-institucional-deinternacionalizacao-2013-capes-print).

9 CAPES. Mudanças na ficha de avaliação valorizam qualidade dos programas. 2019. Disponível em: http://uab.capes.gov.br/36-noticias/9370-mudancas-na-ficha-de-avaliacao-valorizam-qualidade-dos-programas. Acesso em: 10.out 2020.
} 
ou explícito nesses documentos, foi realizada uma busca em documentos de área no site da CAPES ( https://uab.capes.gov.br/avaliacao/sobre-as-areas-de-avaliacao), o qual as divide em três grandes colégios: Ciências da Vida; Humanidades; Ciências Exatas, Tecnológicas e Multidisciplinar. Por sua vez, esses colégios se subdividem, como segue:

\section{CIÊNCIAS AGRÁRIAS Ciência de Alimentos Ciências Agrárias I Medicina Veterinária Zootecnia/Recursos Pesqueiros}

Quadro 1: Ciências da vida

\begin{tabular}{|l|l|}
\hline CIÊNCIAS BIOLÓGICAS & CIÊNCIAS DA SAÚDE \\
\hline Biodiversidade & Educação Física \\
\hline Ciências Biológicas I, II e III & Enfermagem \\
& Farmácia \\
\hline & Medicina I, II e III
\end{tabular}

Nutrição

Odontologia

Saúde Coletiva

Fonte: https://uab.capes.gov.br/avaliacao/sobre-as-areas-de-avaliacao.

\begin{tabular}{|c|c|c|}
\hline CIÊNCIAS HUMANAS & $\begin{array}{l}\text { CIÊNCIAS } \\
\text { APLICADAS }\end{array}$ & $\begin{array}{l}\text { LINGUÍSTICA, LETRAS E } \\
\text { ARTES }\end{array}$ \\
\hline Antropologia/Arqueologia & $\begin{array}{l}\text { Administração Pública e de } \\
\text { Empresas, Ciências Contábeis } \\
\text { e Turismo }\end{array}$ & Artes \\
\hline $\begin{array}{l}\text { Ciência Política e } \\
\text { Relações Internacionais }\end{array}$ & $\begin{array}{l}\text { Arquitetura, Urbanismo e } \\
\text { Design }\end{array}$ & Linguística e Literatura \\
\hline $\begin{array}{l}\text { Ciências da Religião e } \\
\text { Teologia }\end{array}$ & Comunicação e Informação & \\
\hline Educação & Direito & \\
\hline Filosofia & Economia & \\
\hline Geografia & $\begin{array}{l}\text { Planejamento Urbano e } \\
\text { Regional / Demografia }\end{array}$ & \\
\hline História & Serviço Social & \\
\hline Psicologia & & \\
\hline Sociologia & & \\
\hline
\end{tabular}

Fonte: https://uab.capes.gov.br/avaliacao/sobre-as-areas-de-avaliacao.

Quadro 3: Ciências exatas, tecnológicas e multidisciplinar

\begin{tabular}{|l|l|l|}
\hline \multicolumn{1}{c|}{$\begin{array}{c}\text { CIÊNCIAS EXATAS E } \\
\text { DA TERRA }\end{array}$} & \multicolumn{1}{c|}{ ENGENHARIAS } & MULTIDISCIPLINAR \\
\hline Astronomia / Física & Engenharias I & Biotecnologia \\
\hline $\begin{array}{l}\text { Ciência da Computação } \\
\text { Geociências }\end{array}$ & $\begin{array}{l}\text { Engenharias II } \\
\text { Engenharias III }\end{array}$ & Ciências Ambientais \\
\hline $\begin{array}{l}\text { Matemática / } \\
\text { Probabilidade e } \\
\text { Estatística }\end{array}$ & Engenharias IV & Ensino \\
\hline Química & & Interdisciplinar \\
\hline
\end{tabular}

Fonte: https://uab.capes.gov.br/avaliacao/sobre-as-areas-de-avaliacao. 
A consulta foi realizada no período de 20 de junho e 10 de julho de 2020, a partir dos novos documentos de área referentes ao ano de 2019. Os documentos foram lidos e foram destacados os indicadores de internacionalização apontados na seção Perspectivas do processo de internacionalização dos PPGs._Os trechos relevantes, isto é, os que diziam respeito ao uso de línguas, foram agrupados em três categorias e dispostos em uma planilha em Excel:

a) Menção explícita ${ }_{\llcorner}$quando o documento indicava claramente aulas ministradas em inglês (e.g. Ciências Agrárias I: "um conjunto de iniciativas em que a língua inglesa esteja presente nas disciplinas, nas discussões, nos convênios e na mobilidade de autoridades mundiais, além da atração de estudantes internacionais de países desenvolvidos".)

b) Menção implícita, quando o documento indicava aulas em línguas estrangeiras (e.g. Engenharia II: "Participação de pesquisadores visitantes internacionais nas atividades presenciais do programa, incluindo oferta de disciplinas em outro idioma".)

c) Outras ações, quando aulas não eram explicitamente mencionadas (e.g. Geociências: "As publicações dos docentes permanentes devem se dar dominantemente em periódicos internacionais dos estratos mais elevados do Qualis, livros e capítulos publicados no exterior em editoras qualificadas e livros nacionais de alta qualidade; participação de docentes em atividades internacionais".)

\section{IMI nos documentos de área}

Nesta seção, serão trazidas as informações compiladas dos documentos de área de acordo com sua distribuição pelos Colégios e sintetizadas nos quadros 4, 5 e 6 constantes dos Anexos.

O Quadro 4 (Apêndice I) traz os resultados para o Colégio de Ciências da Vida. Das 16 áreas de conhecimento, 5 mencionam explicitamente aulas em inglês (Ciência de Alimentos, Ciências Agrárias I, Medicina Veterinária, Farmácia, Medicina I), 6 fazem referência implícita, ao considerar importante aulas dadas em língua estrangeira, dadas por professores estrangeiros ou mesmo por disciplinas e cursos dados no Exterior (Zootecnia e Recursos Pesqueiros, Educação Física, Fisioterapia, Fonoaudiologia e Terapia Ocupacional, Enfermagem, Medicina II, Nutrição). Nas outras cinco áreas, 
Inglês como meio de instrução na pós-graduação stricto sensu no Brasil: análise dos documentos de áreas

embora não haja referência explícita sobre ensino em inglês, infere-se a valoração maior dada a essa língua, pela menção a qualidade exemplificada por parceria com universidades britânicas (Biodiversidade) ou com foco em publicações internacionais em parceria com pesquisadores internacionais (Ciências Biológicas I e II, Medicina III, Odontologia).

O Quadro 5 (Apêndice II) traz os resultados para o Colégio de Humanidades. Das 20 áreas do conhecimento, 1 (Filosofia) faz menção explícita a aulas em inglês. 5 indicam implicitamente essa ação, seja por mencionar aulas em línguas estrangeiras (Ciências da Religião e Teologia, Educação), seja por prever aulas dadas por professores estrangeiros ou por docentes do programa no Exterior (Comunicação e Informação, Direito, Linguística e Literatura). As demais 14 áreas preveem ações no Brasil e no Exterior, com vários indicadores que incluem publicações internacionais, projetos conjuntos, acordos de cotutela, etc.

O Quadro 6 (Apêndice III) traz os resultados para o Colégio de Ciências Exatas, Tecnológicas e Multidisciplinar. Das 14 áreas de conhecimento, 2 mencionam especificamente o ensino em inglês (Astronomia/Física, Materiais), 4 fazem referência implícita ao mencionar ensino em outras línguas (Química, Engenharias II, Engenharias IV, Ciências Ambientais). As 8 restantes valorizam inserção internacional por meio de publicações e participação em diversas atividades no Exterior ou cooperação internacional, bem como atração de estudantes estrangeiros (Ciência da Computação, Geociências, Engenharias I, Engenharias III, Biotecnologia, Ensino, Interdisciplinar, Matemática/Probabilidade e Estatística).

O Gráfico 1 mostra essas características em percentuais, onde se nota que o Colégio de Ciências da Vida apresenta maior número de áreas com menção explícita a aulas dadas em inglês como indicador de internacionalização, ao passo que o Colégio de Humanidades tem preponderância de outras ações não ligadas necessariamente a aulas em inglês ou em outras disciplinas. De fato, é nesse Colégio que encontramos referências a relações Sul-Sul ${ }^{10}$ como importante estratégia de internacionalização, ao passo que 0 Colégio de Ciências da Vida valoriza publicações em periódicos de alto impacto. Isto

\footnotetext{
10 As relações Sul-Sul se caracterizam pela busca de colaboração com países emergentes, buscando maior simetria entre os países. Distingue-se das relações Norte-Sul, marcadas por hierarquias que subordinam países como o Brasil, em relação a países mais desenvolvidos
} 
sugere que diferentes áreas do conhecimento têm aderido mais fortemente à proposta de que IMI é uma das principais estratégias para atração de discentes e pesquisadores internacionais, assim como para desenvolvimento de proficiência nessa língua, para fins acadêmicos.

Gráfico 1: IMI nos documentos de área da CAPES

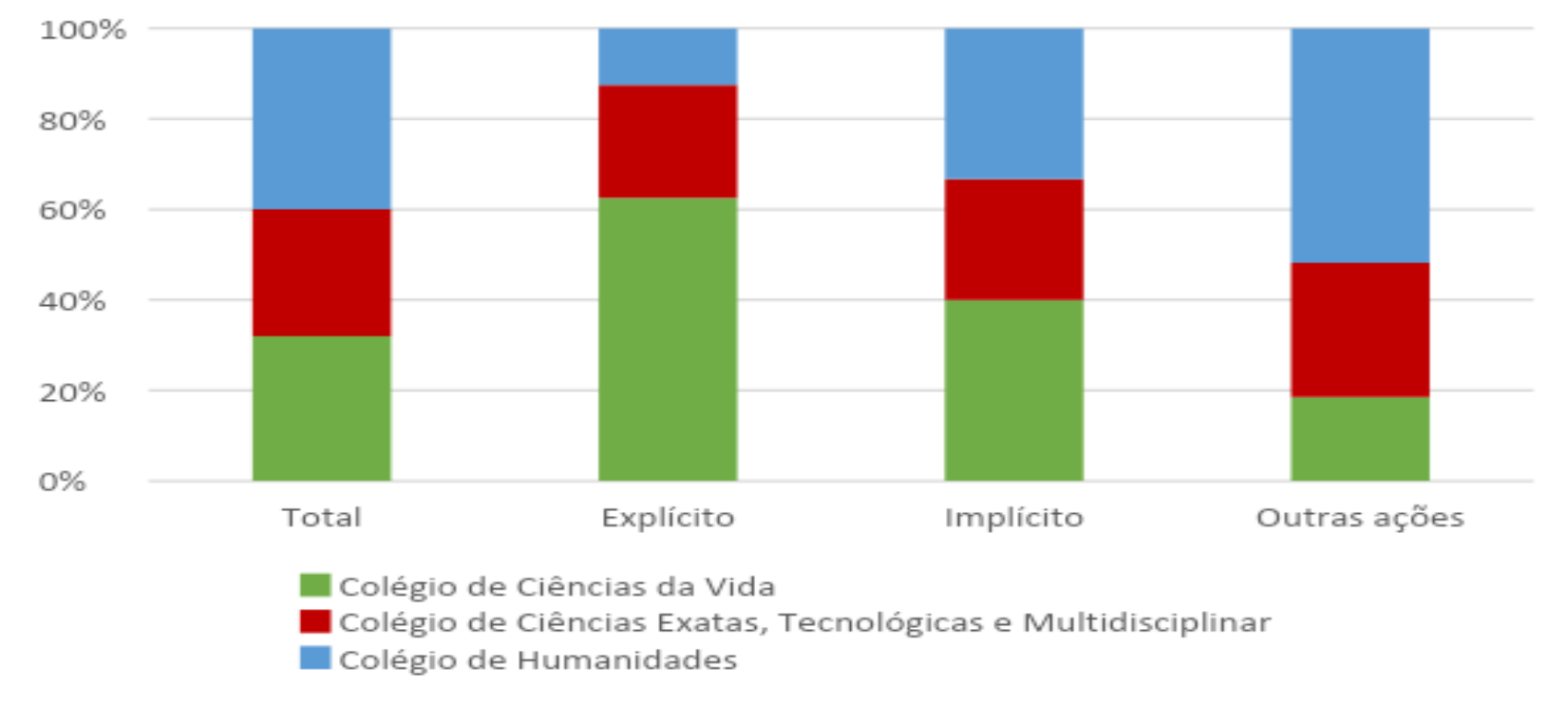

Fonte: Os autores.

\section{Discussão dos resultados}

A maior explicitude em relação a aulas em inglês como indicadores de internacionalização no Colégio de Ciências da Vida indica a importância que essa língua adquire para as áreas do conhecimento que tratam de estudos com maior potencial de se beneficiaram de cooperação internacional, por seu alto grau de generalização. Por outro lado, o Colégio de Humanidades, com áreas que reconhecem o caráter localizado de suas pesquisas, considera outros indicadores como mais significativos (por exemplo, publicações e cooperação internacional).

O caráter implícito, ou seja, a menção a aulas em língua estrangeira, sem listar quais seriam as línguas, pode justificar grande parte de docentes percebendo "cobrança" para dar aulas em inglês, por ser essa língua hegemônica, conforme indicado na introdução deste artigo. Além disso, diversas atividades, como aulas no exterior, podem ser interpretadas como atividades que requerem docência em inglês, o que reforça percepções de que a internacionalização do ensino superior não pode prescindir da língua inglesa.

O reconhecimento explícito do inglês como indicador de internacionalização nos 
Inglês como meio de instrução na pós-graduação stricto sensu no Brasil: análise dos documentos de áreas

programas de pós-graduação pode significar que as áreas dos demais Colégios poderão se ver pressionadas para adotar semelhante critério para ter acesso ao financiamento proporcionado pela agência financiadora. Atualmente, o Colégio de Humanidades é o que tem se inclinado menos para essa tendência, abrindo-se para o multilinguismo e valorizando também relações Sul-Sul, como faz a área de Antropologia/Arqueologia.

A construção do discurso sobre a importância da língua inglesa para a internacionalização inclui a sua incorporação explícita ou implícita em diretrizes de avaliação da qualidade da pós-graduação. Ao fazer esse reconhecimento formal em documentos de área, a comunidade científica brasileira se apropria desse discurso e o fortalece, tornando mais difícil ampliar a diversidade de entendimentos sobre qualidade de pesquisas ou de ensino na pós-graduação. O consenso parece estar sendo desafiado, ainda que timidamente, na área de Humanidades.

\section{Considerações finais}

Este estudo apresentou uma análise de documentos de áreas do conhecimento, produzidos para guiar o processo de avaliação de programas de pós-graduação no Brasil, como possível contribuição para entendimento das motivações e justificativas para adoção do inglês como meio de instrução. Diferentes áreas listam este critério de modo explícito ou implícito, e planejamentos estratégicos institucionais poderão direcionar esforços para atendimento desse requisito, considerando a importância do financiamento a partir do processo de avaliação por pares e configurado nesses documentos. Tal ação é importante, pois "pouco se problematizam as motivações e consequências sociais e educacionais do processo" (JORDÃO et al., 2020, p. 41).

Os resultados deste levantamento preliminar poderão ser complementados por análises mais detalhadas e de cunho qualitativo, junto a docentes, buscando verificar de que modo os implícitos da avaliação são percebidos e quanto estes direcionam suas práticas docentes em contextos de grande competitividade por recursos.

Como sinalizado pelo estudo realizado pelo British Council/Faubai (GIMENEZ et al., 2018), tem crescido o número de disciplinas ministradas em inglês na pós-graduação. Nos próximos anos, poderemos verificar um número ainda maior, se a docência em outra língua que não o português for considerado um indicador importante de internacionalização, reforçada pela percepção de que ela contribui para o 
GIMENEZ, T. N.; OLIVEIRA, H. R.; CARNEIRO, L. A.

desenvolvimento de competências na língua das publicações científicas, quesito valorizado por todas as áreas do conhecimento.

A metáfora de que IMI é um trem em disparada, cunhada por Macaro (2015), tem sido reforçada pelo seu crescimento ao redor do mundo, porém não sem contestação (ver, por exemplo, JORDÃO et al., 2020). Como procuramos mostrar neste estudo, critérios definidores de qualidade na pós-graduação podem estar sendo responsáveis pelo combustível da locomotiva.

\section{Referências}

AERDEN, Axel. The Guide to quality internationalisation. $2^{\text {nd }}$ ed. [S. I.]: ECA Occasional Paper, 2017. Disponível em: http://ecahe.eu/assets/uploads/2013/11/CeQuint-TheGuide-to-Quality-in-Internationalisation-edition-2017-1.pdf. Acesso em: 13 fev. 2021.

BAUMVOL, Laura Knijnik. O uso do inglês como meio de instrução no contexto do ensino superior brasileiro: percepções de docentes. In: Colóquio de Linguística, Literatura e Escrita Criativa [Des]limiares da Linguagem, 9., 2016, Porto Alegre. Anais eletrônicos [...]. Porto Alegre: PUC-RS, 2016, p. 362-370. Disponível em: https://ebooks.pucrs.br/edipucrs/anais/coloquio-de-linguistica-literatura-e-escritacriativa/2016/assets/34.pdf. Acesso em: 13 fev. 2021.

BAUMVOL, Laura Knijnik; SARMENTO, Simone. Can the use of English as a medium of instruction promote a more inclusive and equitable higher education in Brazil? Simon Fraser University Educational Review, Burnaby, v. 12, n. 2, p. 86-105, 2019a.

BAUMVOL, Laura Knijnik; SARMENTO, Simone. The growing presence of language issues in internationalization conferences. In: FINARDI, Kyria Rebeca. (org.). English in the South. Londrina: EDUEL, 2019b, p. 27-54.

BAUMVOL, Laura Knijnik; SARMENTO, Simone. A internacionalização em casa e o inglês como meio de instrução. In: BECK, Magali Sperling et al. ECHOES: Further Reflections on Language and Literature. Florianopolis: EdUFSC, 2016, p. 65-82.

BRASIL. Ministério da Educação. Mudanças na ficha de avaliação valorizam qualidade dos programas. 2019. Disponível em: https://www.gov.br/capes/ptbr/assuntos/noticias/mudancas-na-ficha-de-avaliacao-valorizam-qualidade-dosprogramas ramas. Acesso em: 10 out. 2020.

BRASIL. Ministério da Educação. Presidente explica importância da avaliação para a pós-graduação. Brasília: MEC, 2020a. Disponível em: https://www1.capes.gov.br/36noticias/10389-presidente-explica-importancia-da-avaliacao-para-a-pos-graduacao. Acesso em: 13 fev. 2021.

BRASIL. Ministério da Educação. Sobre as áreas de avaliação. Brasília: MEC, 2020b. Disponível em: https://www.gov.br/capes/pt-br/acesso-a-informacao/acoes-eprogramas/avaliacao/sobre-a-avaliacao/areas-avaliacao/sobre-as-areas-de- 
Inglês como meio de instrução na pós-graduação stricto sensu no Brasil: análise dos documentos de áreas

avaliacao/sobre-as-areas-de-avaliacao\#areas. Acesso em: 13 fev. 2021.

BRITISH COUNCIL. Do ensino básico à universidade: pesquisas e colaborações sobre a língua inglesa: UK-Brazil english collaboration call 2018-2019. 2020. Disponível em: https://www.britishcouncil.org.br/atividades/educacao/internacionalizacao/uk-brazilenglish-collaboration-call. Acesso em: 24 jul. 2020.

BRITISH COUNCIL. Guide of Brazilian Higher Education Courses in English 2016. 2016. Disponível em:

https://www.britishcouncil.org.br/sites/default/files/guide_brazilian_highered_courses_i nenglish.pdf. Acesso em: 26 jul. 2020.

DEARDEN, Julie. English as a medium of instruction- a growing global phenomenon. London: British Council, 2014. Disponível em: https://ora.ox.ac.uk/objects/uuid:4f72cdf8-b2eb-4d41-a7854 a283bf6caaa/download_file?file_format=pdf\&safe_filename=EMI\%2Ba\%2BGrowing\%2BGlobal\%2BPhenomenon.pdf\&type_of_work=Report. Acesso em: 20 jun. 2020.

DOIZ, Aintzane; LASAGABASTER, David; SIERRA, Juan Manuel (ed.). English-Medium instruction at universities: global challenges. Bristol: Multilingual Matters, 2013.

FINARDI, Kyria Rebeca; GUIMARÃES, Felipe Furtado. Internacionalização, rankings e publicações em inglês: a situação do Brasil na atualidade. Estudos em Avaliação Educacional. São Paulo, v. 28, n. 68, p. 600-626, maio/ago. 2017.

GIMENEZ, Telma et al. Guide to English as a medium of instruction in Brazilian Higher Education institutions 2018-2019. [S. I.]: FAUBAI, 2018. Disponível em:

http://faubai.org.br/britishcouncilfaubaiguide2018.pdf. Acesso em: 26 jul. 2020.

JENKINS, Jennifer. English as a lingua franca in the international university. London: Routledge, 2014.

JORDÃO, Clarissa; FIGUEIREDO, Eduardo Henrique Diniz; LAUFER, Gabriela Fagundes; FRANKIW, Thaina Caroline. Internacionalização em inglês: sobre esse tal de unstoppable train e de como abordar a sua locomotiva. Revista $\tilde{N} E M I T Y \bar{R} \tilde{A}$, v. 1, n.2, p. 30-43, 2020. Disponível em:

https://drive.google.com/file/d/1A2wH5m_TsboXXV6UoZHWTGKZna5mgKI8/view . Acesso em: 15 fev. 2021.

KIRKPATRICK, Andy. Internationalization or Englishization: medium of instruction in today's universities. Hong Kong: Hong Kong Institute of Education, 2011. Disponível em: http://repository.lib.ied.edu.hk/pubdata/ir/link/pub/201705439.pdf. Acesso em: 10 fev. 2021.

MACARO, Ernesto. English medium instruction: time to start asking some difficult questions. Modern English Teacher, v. 24, n.2, 2015.

MACARO, Ernesto; CURLE, Samantha; PUN, Jack; AN, Jiangshan; DEARDEN, Julie. A 
systematic review of English medium instruction in higher education. Language Teaching, v. 51, n.1, p. 36-76, 2018.

MARTINEZ, Ron. English as a Medium of Instruction (EMI) in Brazilian higher education: challenges and opportunities. In: FINARDI, Kyria Rebeca (org.). English in Brazil: views, policies and programs. Londrina: EdUEL, 2016. p. 193-228.

MARTINEZ, Ron; FERNANDES, Karina. Development of a Teacher Training Course for English Medium Instruction for Higher Education Professors in Brazil. In: SÁNCHEZPÉREZ, María del Mar. Teacher training for English-medium instruction in higher education. [S. I.]: IGI Global, 2020. p. 125-152.

MURATA, Kumiko. English-Medium Instruction from an English as a Lingua Franca perspective: exploring the higher education context. Abingdon: Routledge, 2018.

MURATA, Kumiko; LINO, M. EMI in higher education: an ELF perspective. In: JENKINS, Jennifer; BAKER, Will; DEWEY, Martin. (org.). The Routledge Handbook of English as a Lingua Franca. Abingdon: Routledge, 2018, p. 400-412.

RIZVI, Fazal; LINGARD, Bob. Globalizing education policy. London: Routledge, 2010.

VERDU, Fabiane Cortez. EMI (English as a medium of instruction) como estratégia de internacionalização em casa: um estudo de caso num programa de pós-graduação em administração. In: ENCONTRO DA ANPAD - EnANPAD, 41., 2017, Maringá. Anais [...]. Maringá: UEM, 2017, p. 01-08.

WIT, Hans de. Internationalisation of higher education in Europe and its assessment, trends, and issues. Nederlands: The Accreditation Organisation of the Netherlands and Flanders, 2010. Disponível em: https://www.eurashe.eu/library/modernisingPhe/mobility/internationalisation/WG4\%20R\%20Hans\%20de\%20Wit\%20Internationalis ation_of_Higher_Education_in_Europe_DEF_december_2010.pdf. Acesso em: $12 \mathrm{dez}$. 2020.

ZÜGE, Aline Priscilla Brancalhão; BARRETO, Ana Igraíne de Góis; NOVELLI, Josimayre. EMI em foco: percepções, possibilidades e desafios. Revista NUPEM, Campo Mourão, v. 12, n. 26, p. 43-61, 2020. 